\title{
A novel radiant heat test for assessing pain threshold in human subjects: Measurement stability
}

\author{
JANA HERRMAN LEE and MAXINE L. STITZER \\ Johns Hopkins University School of Medicine, Baltimore, Maryland
}

\begin{abstract}
New methodologies to assess analgesic response in humans are needed to better integrate preclinical and clinical data. In the present study we examined the test-retest stability of an innovative radiant heat methodology compared with an electrical stimulation methodology. For the radiant heat task, a modified rodent tail flick apparatus was used. The latency for finger withdrawal was recorded. For the electrical stimulation tasks, subjects placed two fingers on two electrodes from which they received a brief series of increasingly intense electrical stimulations. Maximum stimulus intensity (in milliamps) delivered was recorded. On each of 4 test days, the subjects received five test trials with a 10-min interval between trials. All the subjects were tested twice on each apparatus in a counterbalanced design. Finger withdrawal latencies for the radiant heat task did not differ significantly across test trials or test days. Finger withdrawal scores for electrical stimulation increased significantly across test trials as well as test days. These data show that the radiant heat method generates consistent latencies across trials and days, whereas shock produces trends over time. The radiant heat task, which is convenient to operate and inexpensive to build, appears promising as a reliable test of pain threshold in humans.
\end{abstract}

Many attempts have been made to develop a simple and reliable laboratory method to assess pain thresholds in human volunteers. Some of these methods include ice water hand immersion, thermal heat, electrical stimulation, and skin pressure tests (Chéry-Croze, 1983; Collier, Mehta, \& Spear, 1990; Harris \& Rollman, 1983). Among these different stimuli, thermal heat and electrical stimulation have been chiefly used to induce experimental pain (Chéry-Croze, 1983). Additionally, these methods have been frequently used in nonclinical studies in which repeated exposure to the stimulus is often required. However, considerable variation in pain threshold values have been reported with electrical stimulation. For instance, Collier et al. reported significant variation in threshold responses following repeated exposure to electrical stimulation. This may be due to a developed tolerance or adaptation that may occur over time to the sensations produced by electrical stimulation. Additionally, Harris and Rollman reported that electrical stimulation is often accompanied by subjective reports of anxiety and stress. Hence, the psychological components associated with electrical stimulation may lead to variability in responding.

Thermal heat tests have been successfully used to determine pain threshold in human volunteers for the last three decades (Hardy, Goodell, \& Wolff, 1952). The two most commonly employed methods of delivering thermal heat are radiant heat (use of a beam of light or laser pulse) and conducted heat (use of Peltier thermodes on

Address correspondence to J. H. Lee, Department of Psychiatry and Behavioral Sciences, Johns Hopkins/Key Medical Center, 4940 Eastern Avenue, Baltimore, MD 21224. the skin). Both methods have produced reliable threshold responses in human volunteers (Croze \& Duclaux, 1978; Yarnitsky \& Ochoa, 1990), but they are not without their limitations. For example, some radiant heat devices do not deliver a sharp, localized beam of light. This leads to a greater surface area's being affected by the beam and hence greater variability in responding (Chéry-Croze, 1983). Mor and Carmon (1975) and Meyer, Walker, and Mountcastle (1976) developed a laser pulse technique that is capable of delivering controlled stimuli, both sharply localized and very brief. However, laser pulse devices are extremely costly, which makes widespread use and ultimate replication of results unlikely.

Radiant heat tests, such as the tail flick test, are frequently used in preclinical research. In this test, the latency of the animal to withdraw its tail from the heat source is used as an index of pain sensitivity. The tail flick test has been used to gauge the potency of a variety of narcotic and nonnarcotic agents. Currently, there is no exactly comparable method of assessing pain threshold in human subjects. New methodologies to assess pain threshold are needed to better integrate preclinical and clinical data (Chapman et al., 1985). The purpose of the present study was to test the reliability of an innovative radiant heat methodology, on the basis of methods frequently used in preclinical research, for assessing pain threshold in human volunteers.

\section{METHOD}

\section{Subjects}

Fourteen healthy college students ( 12 females and 2 males) between the ages of 18 and $29(M=20.5 \pm .8$ years $)$ participated in the study. The subjects were recruited through college newspaper 
advertisements. Each subject received a thorough oral and written explanation of the procedures and objectives of the experiment. and all signed an informed consent form before participation. The procedures used in this study received prior approval from the Johns Hopkins University Medical School Internal Review Board. The subjects were paid $\$ 100$ for completion of the 4-day study, or $\$ 10$ for each completed test session. All the subjects completed the 4-day study.

\section{Design}

We used a within-subject design that compared consistency of pain threshold responses across time. The subjects attended four 1 -h test sessions. During each session, they were exposed to a series of stimulus presentations delivered by either a finger flick light beam apparatus or an electrical stimulation apparatus. Presentation of either radiant heat or electrical stimulation was counterbalanced (i.e., the subjects were randomly assigned to one test condition on Test Day 1 and then alternated between the two test conditions on Test Days 2, 3, and 4). Pain threshold, rather than maximum pain tolerated, was examined because it is less likely to produce tissue damage, and threshold measurements are more consistènt with the preclinical literature.

\section{Procedure}

Finger flick task. A modified tail flick apparatus similar to that described by Walker and Dixon (1983) was used as a finger flick apparatus. In brief, the finger flick device consists of a radiant heat source, photocell, timer, and power source. A black dot was placed on the dominant index finger midway between the fingertip and the distal interphalangeal joint. This procedure allowed for accurate finger placement on the finger flick device for each test trial. The subject was instructed to place his/her finger over a $3-\mathrm{mm}$ hole, through which the light from a projection bulb radiated from below. Above the finger was a photocell; thus, with the finger covering the light source, light was prevented from hitting the photocell. A switch activated the lamp and the timer. When the subject removed his/her finger, the light hit the photocell, automatically stopping the timer and turning off the light. Each subject received five test trials with a 10-min delay between trials. Waiting at least 10 -min between each trial prevented the subjects from becoming habituated to the stimulus. The subjects were also instructed to remove the finger once the stimulation started to become painful. The subjects were instructed to focus on a dot placed on the wall directly in front of them. This prevented them from focusing on the light radiating from the apparatus. The latency for finger withdrawal was recorded.

Electrical stimulation task. The electrical stimulation was induced with a Grass Model S48 stimulator. A constant current and a duration of $0.6 \mathrm{sec}$ was used. The voltage setting on the stimulator was held constant at 16 units, and the multiplier was variable from 1 to 10 . The electrical stimulation was delivered to the fingertips of the dominant middle and index fingers. The subjects were instructed to place their fingertips on top of two table-mounted silver electrodes. The initial current applied was below sensation threshold $(0.25 \mathrm{~mA}, 0.6-\mathrm{sec}$ duration); the current was then increased by $0.5 \mathrm{~mA}$ for each successive stimulus presentation, with pulses delivered once every $5 \mathrm{sec}$ until the subject terminated the test trial. Each subject received five test trials with a 10 -min delay between trials. The subjects were instructed to remove their fingers from the silver electrodes once the stimulation started to become painful. Maximum stimulus intensity (in milliamps) delivered was recorded.

Subjective assessment. Immediately following each determination of pain threshold, the subjects marked visual analog scales $(0-10$ points) relating stimulus intensity (how painful), as well as a variety of stimulus characteristics, including unpleasantness, burning, and tingling of the preceding test.
Physical measurements. Finger temperature was measured prior to each trial, and a heating pad was placed on the hand if the temperature dropped more than $5^{\circ}$. Individual finger temperature was monitored and held within $5^{\circ}$ of Trial 1 finger temperature throughout the test session; pilot testing indicated that severe fluctuations in finger temperature altered threshold responses for the radiant heat test. Swings in finger temperature did not appear to alter responses to electrical stimulation in our pilot population.

Data analysis. Threshold response measures were analyzed separately for each condition with two-way repeated measures analyses of variance (ANOVAs) to examine time trends across test days and test trials within days. Response consistency was further evaluated by using correlation statistics to determine test-retest reliabilities for each measure. Measures of subjective ratings and finger temperature were analyzed with three-way repeated measures ANOVAs to examine effects of test condition (heat vs. electrical stimulation), time trends across test days, and test trials within days and test conditions. All results are presented as means \pm SEM (standard error of the mean).

\section{RESULTS}

Figure 1 shows the maximum milliamps tolerated for electrical stimulation and finger withdrawal latencies for the radiant heat test. Mean finger withdrawal latencies collapsed across all days and trials were $7.6 \pm .3 \mathrm{sec}$.
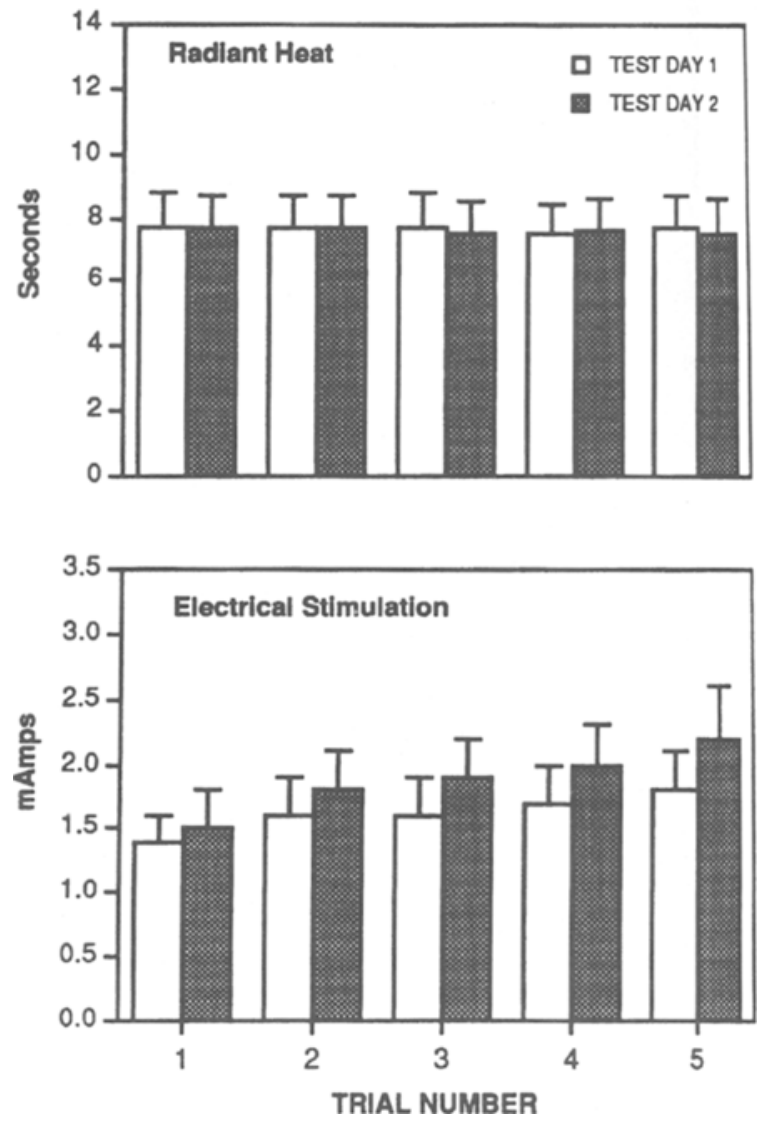

Figure 1. Maximum milliamps tolerated for electrical stimulation and finger withdrawal latencies (seconds) for the radiant heat test. Values are means $\pm S E M$ (standard error of the mean). 
Latencies remained stable across test trials $[F(4,52)=$ $2.92, p>.1]$ and test days $[F(4,52)=.57, p>.1]$. Mean milliamps tolerated ranged from $1.4 \mathrm{~mA}$ on Trial 1 , Day 1 to $2.2 \mathrm{~mA}$ on Trial 5, Day 2. There was a significant trial effect for electrical stimulation $[F(4,52)=$ $6.07, p<.01$; see Figure 1] and a trend for higher withdrawal scores on Day $2[F(4,52)=2.94, p>.1]$.

Test-retest (Day 1 vs. Day 2) correlations were performed on the mean test trial score (collapsed over the five trials) for each subject. Test-retest correlation coefficients were significant for both electrical stimulation and radiant heat $(r=.88, p<.01$ and $r=.95, p<.01$, respectively; see Figure 2). Time trends for electrical stimulation shown in Figure 1 are reflected in the slightly lower correlation and greater variability around the regression line.

Overall subjective ratings of painfulness did not differ significantly for the two tests $[F(4,52)=3.33, p>.1]$. However, subjective ratings of painfulness tended to in-
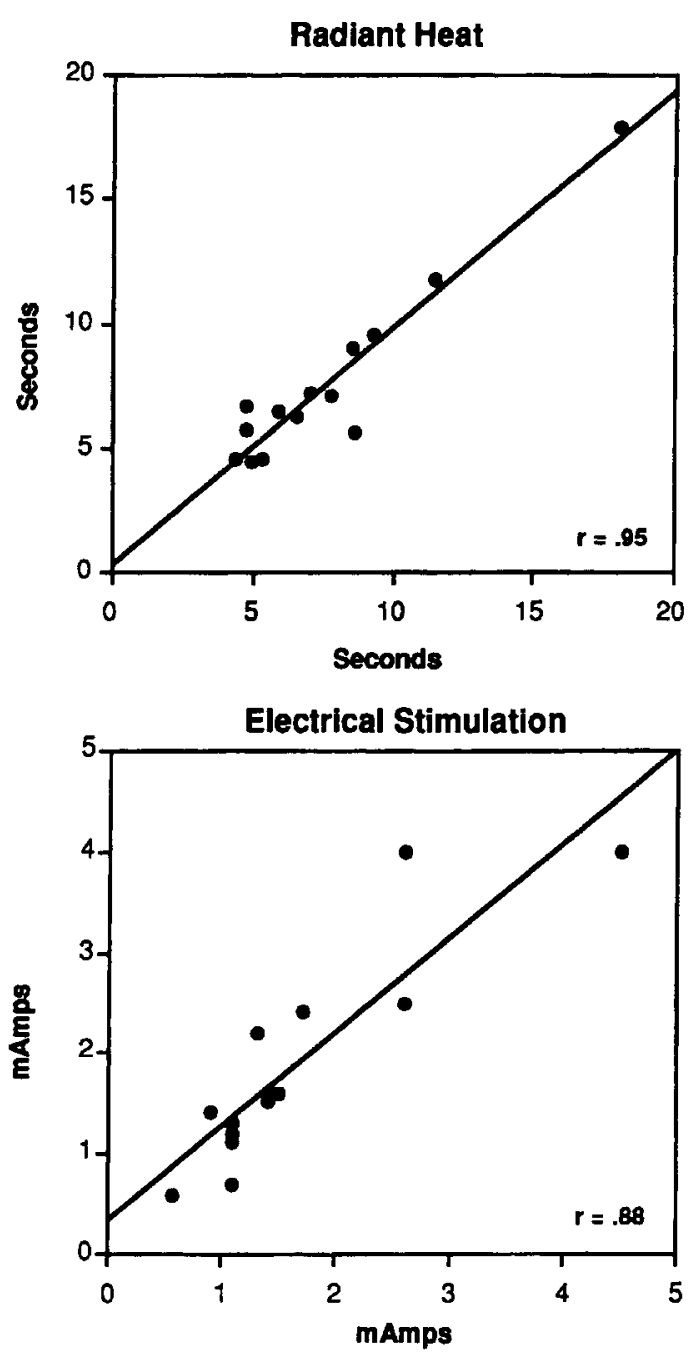

Figure 2. Test-retest (Day 1 vs. Day 2) correlations for mean test trial scores (collapsed over the five trials) for radiant heat and electrical stimulation.
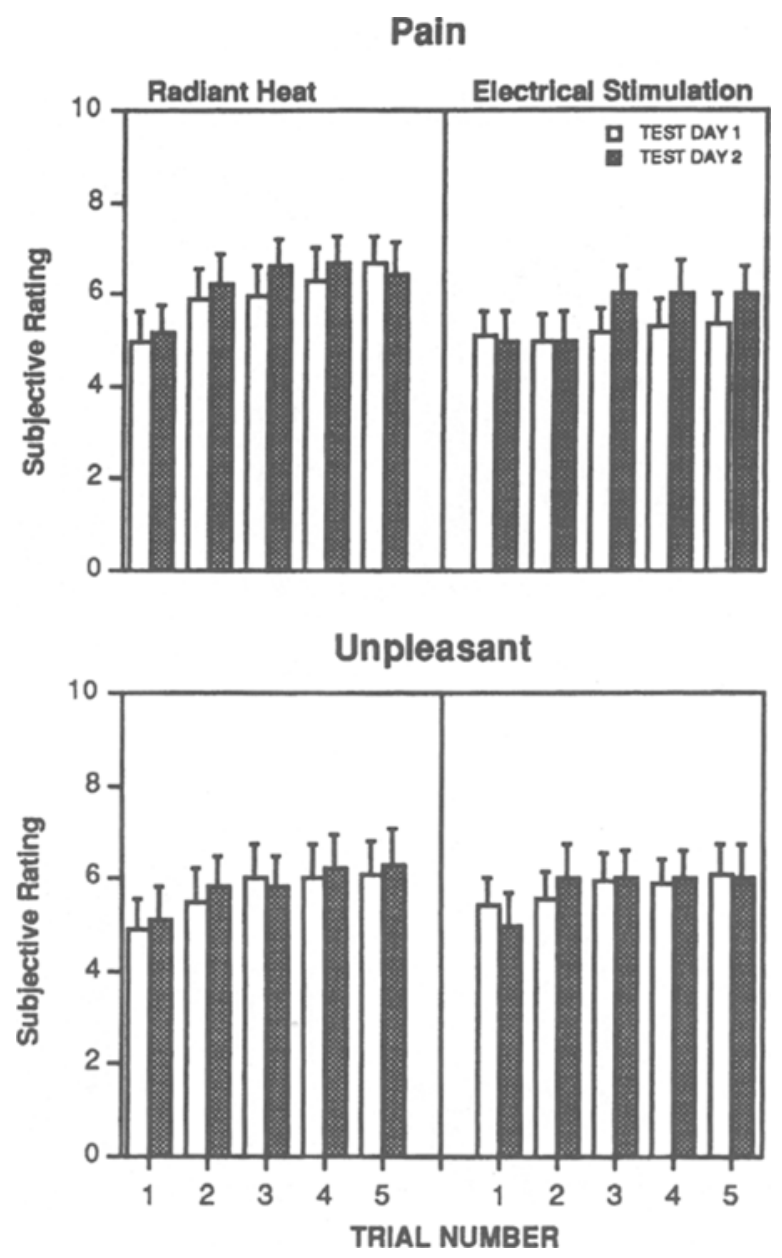

Figure 3. Subjective ratings of painfulness and unpleasantness for radiant heat and electrical stimulation. Values are means $\pm S E M$ (standard error of the mean).

crease across test trials for the radiant heat test $[F(4,52)=$ $5.25, p<.05]$. As can be seen in Figure 3, this is primarily due to the lower score on the first trial. Ratings of unpleasantness were similar for both radiant heat and electrical stimulation $[F(4,52)=.90, p>.1]$. Unpleasantness ratings increased significantly across test trials for both radiant heat and electrical stimulation $[F(4,52)=$ $13.24, p<.005]$. As is shown in Figure 3, this is primarily due to lower scores on the first trial.

Figure 4 shows the qualitative ratings of the stimuli. As expected, the subjects rated the radiant heat test as significantly more burning than the electrical stimulation test $[F(4,52)=11.40, p<.005$; see Figure 4]. Subjective ratings for tingling were significantly higher for the electrical stimulation test than for the radiant heat test $[F(4,52)=3.40, p<.05$; see Figure 4$]$. The only time trend was a significant trial effect for burning $[F(4,52)=$ $30.26, p<.005]$.

Finger temperature was measured prior to each test trial. There were no condition effects $[F(4,52)=1.74$, $p>.1]$. Average finger temperature collapsed across all 

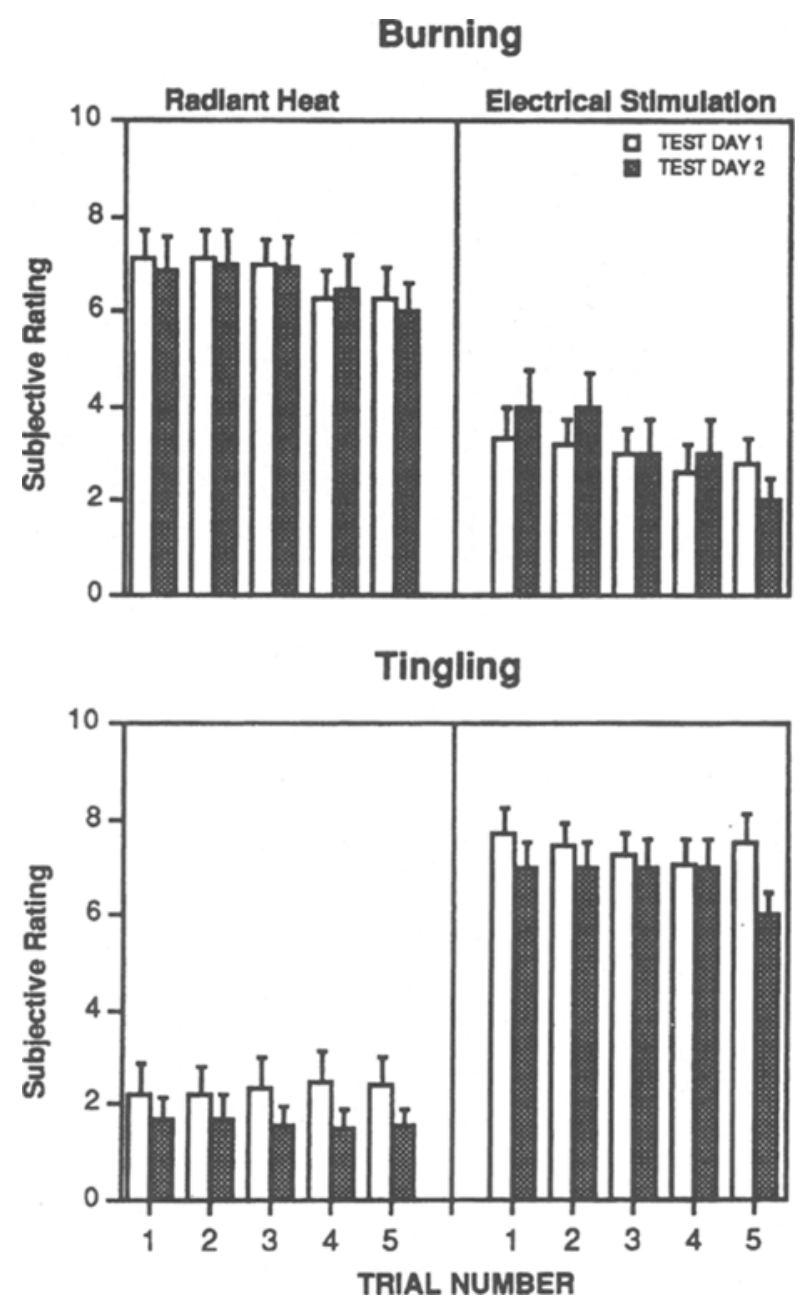

Figure 4. Qualitative ratings of burning and tingling for the radiant heat and electrical stimulation tasks. Values are means $\pm S E M$ (standard error of the mean).

trials for both conditions was $30.6^{\circ} \mathrm{C} \pm .2^{\circ}$. Finger temperature tended to be slightly lower $\left(M=28.5^{\circ} \mathrm{C} \pm .3^{\circ}\right)$ on the first trial than on subsequent trials in both conditions $[F(4,52)=27.50, p<.05]$.

\section{DISCUSSION}

The purpose of this study was to test the reliability of an innovative radiant heat methodology for assessing pain threshold in human volunteers on the basis of methods frequently used in preclinical research. Our radiant heat test is comparable to the tail flick methodology frequently used in preclinical research. Specifically, the subjects were instructed to place a finger over a hole on the apparatus and to remove it as soon as the heat stimulation started to become painful (presumably when a rodent would withdraw its tail).

The radiant heat methodology described here produces extremely consistent finger withdrawal latencies across test days and within test sessions. Recently, Per- kins, Grobe, Jennings, Epstein, and Elash (1992) reported similar findings using a radiant heat methodology focused on the leg. They found consistent pain threshold reports within test sessions; however, their radiant heat methodology showed more variability across test days. The present methodology produced stable finger withdrawal latencies across a 2 -week period. Hence, this methodology would be ideal for protocols that required (1) repeated pain threshold response measurements in one test session, and (2) repeated measurements across several test days. In contrast, the electrical stimulation test described here resulted in significant increases in total milliamps tolerated within each test session. Although these might stabilize over time, there is an advantage to having a measure that is stable right away. There are several negative implications to the findings with electrical stimulation. For example, if this type of methodology were to be used to assess the analgesic efficacy of certain drugs, the test-retest drift would result in increased pain thresholds regardless of drug effects.

Overall subjective ratings of painfulness did not differ for the two methodologies. However, there was a trend for the subjective ratings of painfulness to be slightly lower on the first trial for the radiant heat test. This may be due to some expectancy effects, the subject getting used to the protocol and the questionnaires, or the slightly lower finger temperature. Subjective ratings of unpleasantness again did not differ for the two methodologies. However, at the debriefing, the subjects were asked which method they found to be more painful and which more unpleasant. Interestingly, $65 \%$ selected the radiant heat as more painful, while $28 \%$ selected the electric shock as more painful; 1 subject feit that neither was actually painful. On the unpleasant dimension, $100 \%$ claimed that the electrical stimulation test was more unpleasant, and several of the subjects described the electrical stimulation as "aversive," suggesting a negative association with electric shock. Harris and Rollman (1983) found that electrical stimulation was frequently reported to increase subjective ratings of anxiety and stress. These findings are consistent with the present report.

There are several factors that can affect the stability of responding with our radiant heat methodology. Pilot testing indicated that fluctuations in finger temperature can greatly affect response consistency. For example, subjects with lower finger temperatures will generally have longer latencies than subjects with higher finger temperatures. If a subject's finger temperature either rises or falls during a test session, finger latency will in turn either rise or fall. It is extremely important to keep finger temperature constant during test sessions. Another contributing factor is the marking of the finger, which facilitates heat absorption. It has been shown that the faster the stimulus intensity increases, the more reliable the pain threshold responses (Perkins et al., 1992; Yarnitsky \& Ochoa, 1990). Marking the finger also allows for accurate placement of the finger on the apparatus over repeated trials. Finally, it is advisable to wait at 
least 10 min between test trials in order to ensure response consistency. Intervals shorter than $10 \mathrm{~min}$ result in increasingly shorter latencies in rats (Lee \& McCarty, 1990). This may be due to an increasing skin temperature or lack of recovery from stimulation of nerve fibers.

Our radiant heat methodology is practical to implement. The finger flick device was inexpensive to build based on the design described by Walker and Dixon (1983). As mentioned earlier, laser pulse methodologies produce reliable pain threshold responses, yet the high cost of the equipment makes them impractical to most laboratories (Chéry-Croze, 1983). The equipment for the radiant heat method only measures $12.7 \times 25.4 \times$ $7.62 \mathrm{~cm}$, so it does not take up much laboratory space and can be portable. Other radiant heat apparatuses frequently are bulky and difficult to use (Chéry-Croze, 1983). Our device is inexpensive, portable, and easy to operate.

There may be a number of applications of this method to basic behavioral research. Because finger withdrawal latencies remained consistent across test days and within test sessions, this method may be sensitive to acute behavioral or pharmacological manipulations. Radiant heat methodologies have often been criticized as being insensitive to analgesic states (Beecher, 1959; Chapman et al., 1985; Chapman, Dingman, \& Ginzberg, 1965). However, this methodology has been sensitive to glucoseinduced alterations in pain threshold in diabetic animals (Lee \& McCarty, 1990, 1992). Finally, preliminary results on morphine-induced alterations in pain threshold in human volunteers are showing this methodology to be promising.

In summary, the radiant heat methodology described in this report resulted in stable pain threshold responses in human volunteers. In contrast, a comparable electrical stimulation methodology resulted in an increase in withdrawal latencies across test trials and test days.

\section{REFERENCES}

BEECHER, H. K. (1959). Measurement of subjective responses: Quantitative effects of drugs. New York: Oxford University Press.

Chapman, C. R., Casey, K. L., Dubner, R., Foley, K. M., Gracely, R. H., \& Reading, A. E. (1985), Pain measurement: An overview. Pain, 22, 1-13.

Chapman, L. F., Dingman, H. F., \& Ginzberg, S. P. (1965). Failure of systemic analgesic agents to alter the absolute sensory threshold for the simple detection of pain. Brain, 88, 1011-1022.

Chéry-Croze, S. (1983). Painful stimulation induced by a thermal cutaneous stimulus. Pain, 17, 109-137.

Collier, J. L., Mehta, B. M., \& Spear, F. G. (1990). Experimental changes in pain threshold and severe pain threshold for electrically induced pain. Psychosomatic Medicine, 43, 99-102.

Croze, S., \& DuClaux, R. (1978). Thermal pain in humans: Influence of the rate of stimulation. Brain Research, 157, 418-421.

HARDY, J. D., GoOdell, H., \& WolfF, H. G. (1952). Pain sensations and reactions. Baltimore: Williams \& Wilkins.

HaRris, G., \& Rollman, G. B. (1983). The validity of experimental pain measures. Pain, 17, 369-376.

LEE, J. H., \& MCCARTY, R. (1990). Glycemic control of pain threshold in diabetic and control rats. Physiology \& Behavior, 47, 225-230.

LeE, J. H., \& MCCARTY, R. (1992). Pain threshold in diabetic rats: Effects of good versus poor diabetic control. Pain, 50, 231-236.

Meyer, R. A., Walker, R. E., \& Mountcastle, V. B. (1976). A laser stimulator for the study of cutaneous thermal and pain sensations. IEEE Transactions on Biomedical Engineering, 23, 54-60.

MOR, J., \& CARMON, A. (1975). Laser emitted radiant heat for pain research. Pain, 1, 233-237.

Perkins, K. A., Grobe, J. E., Jennings, J. R., Epstein, L. H., \& Elash, E. (1992). A technique for rapid, reliable assessment of thermal-pain threshold in humans. Behavior Research Methods, Instruments, \& Computers, 24, 60-66.

WALKER, J. M., \& DiXON, W. C. (1983). A solid state device for measuring sensitivity to thermal pain. Physiology \& Behavior, 30, 481483.

YARNITSKY, D., \& OCHOA, J. L. (1990). Studies of heat pain sensation in man: Perception thresholds, rate of stimulus rise and reaction time. Pain, 40, 85-91.

(Manuscript received January 11, 1993; revision accepted for publication April 28, 1994.) 